\title{
Isolated acute hemorrhagic cholecystitis in liver cirrhosis after blunt trauma
}

Received October 11, 2021

Revised October 21, 2021

Accepted October 22, 2021

Correspondence to Dong Hun Kim

Department of Trauma Surgery, Trauma Center, Dankook University Hospital, 201 Manghyang-ro, Dongnam-gu,

Cheonan 31116, Korea

Tel: +82-41-550-3042

Fax: +82-41-550-0039

E-mail: saint7331@gmail.com

\author{
Yoonjung Heo, Dong Hun Kim \\ Department of Trauma Surgery, Trauma Center, Dankook University Hospital, Cheonan, \\ Republic of Korea
}

Isolated acute hemorrhagic cholecystitis (AHC) after blunt trauma is extremely rare. Thus, alcoholic liver cirrhosis can be a risk factor for this type of injury. The use of point-of-care ultrasound as a monitoring tool for suspicious gallbladder injuries facilitates rapid recognition and decision-making. Therefore, laparoscopic cholecystectomy was recommended for treating traumatic AHC in patients with hemodynamically stable liver cirrhosis.

Key Words: Gallbladder, Acute cholecystitis, Liver cirrhosis, Nonpenetrating wounds, Abdomen

\section{Introduction}

Gallbladder (GB) injuries from blunt abdominal trauma are rare with a reported incidence of $0.5 \% \pm 0.6 \%$ of all intra-abdominal injuries (1). The isolated GB injuries are even more unusual. Less than $2 \%$ of traumatic GB injuries are without concomitant abdominal pathology (2). A unique case of isolated acute hemorrhagic cholecystitis (AHC) in a patient with alcoholic liver cirrhosis after blunt trauma has been described here.

\section{Case Presentation}

A 42-year-old male was admitted after being run over by a car while lying on the road. The patient's initial hemodynamic parameters were stable (blood pressure: $120 / 80$ $\mathrm{mmHg}$; heart rate: 75 beats per min; temperature: $36.1^{\circ} \mathrm{C}$; saturation: $98 \%$ on room air) with a Glasgow Coma Scale of 6 under the influence of alcohol intake. The patient had a liver cirrhosis (Child-Pugh class A) background with alcohol dependence and panic disorder. A scalp avulsion of $20 \mathrm{~cm}$ with exposure of a compressed skull fracture

Table 1. Laboratory results

\begin{tabular}{lcc}
\hline \multirow{2}{*}{\multicolumn{1}{c}{ Variables }} & \multicolumn{2}{c}{ Values } \\
\cline { 2 - 3 } & HD \#1 & HD \#2 \\
\hline $\mathrm{Hb}(\mathrm{g} / \mathrm{dL})$ & 10.5 & 9.6 \\
$\mathrm{WBC}(/ \mu \mathrm{l})$ & 7290 & 8120 \\
Platelet $(/ \mu \mathrm{l})$ & $141 \mathrm{~K}$ & $50 \mathrm{~K}$ \\
AST $(\mathrm{U} / \mathrm{L})$ & 317 & 758 \\
ALT $(\mathrm{U} / \mathrm{L})$ & 115 & 187 \\
ALP $(\mathrm{U} / \mathrm{L})$ & 89 & 95 \\
Total bilirubin $(\mathrm{mg} / \mathrm{dL})$ & 0.96 & 1.15 \\
GGT $(\mathrm{IU} / \mathrm{L})$ & - & 579 \\
CRP $(\mathrm{mg} / \mathrm{dL})$ & 0.04 & 8.89 \\
\hline
\end{tabular}

HD: hospital day.

(c)This is an Open Access article distributed under the terms of the Creative Commons Attribution Non-Commercial License (http://creativecommons.org/licenses/by-nc/4.0) which permits unrestricted noncommercial use, distribution, and reproduction in any medium, provided the original work is properly cited.

Copyright (C) 2021 Korean Association for Research, Procedures and Education on Trauma. All rights reserved. 
and multiple avulsions of the right anterior chest wall was noted in the primary survey. Abdominal tenderness was uncheckable. Computed tomography (CT) scans showed isolated AHC (Fig. 1) and a minor laceration in the right middle lobe of the lung. The injury severity score was 22 .
The patient became conscious the following day and complained of abdominal pain in the right upper quadrant and positive Murphy's sign. The patient remained hemodynamically stable, but blood tests deteriorated (Table 1). Point-of-care ultrasound (POCUS) obtained with a Vivid
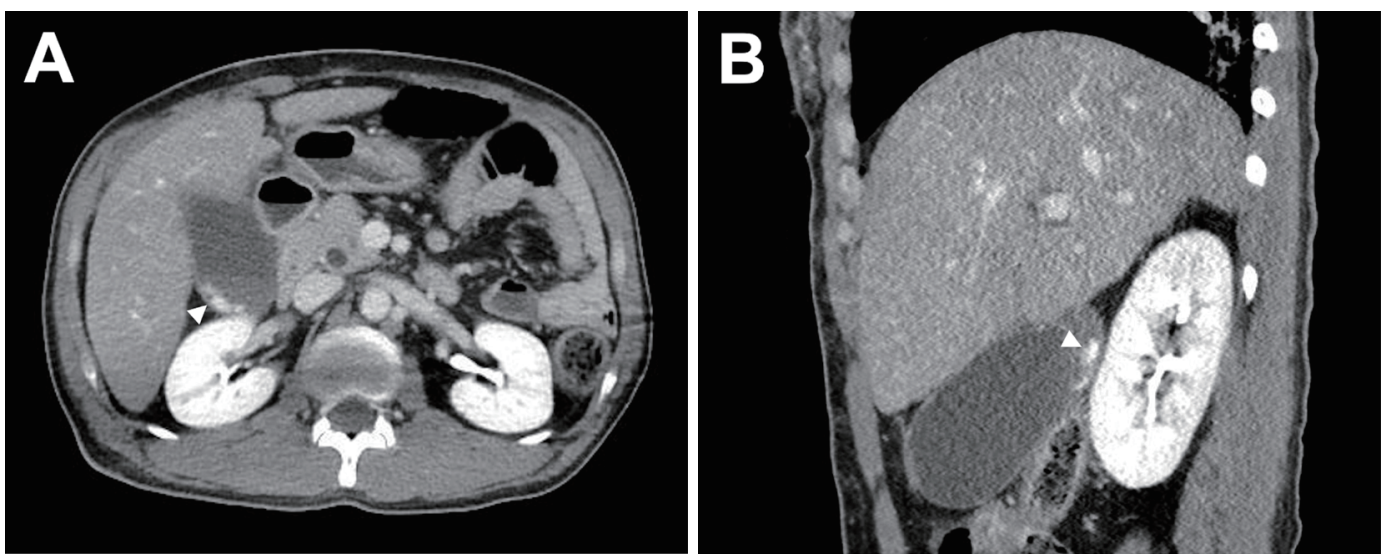

Fig. 1. Axial (A) and sagittal (B) views of the initial abdominal computed tomographic scans show isolated acute hemorrhagic cholecystitis with a dilatated extrahepatic common bile duct and cholelithiasis. Active extravasation of the contrast blush (arrowheads) into the lumen of the distended gallbladder is noted on the portal delayed phase. The liver parenchyma presents cirrhosis-like heterogeneities.
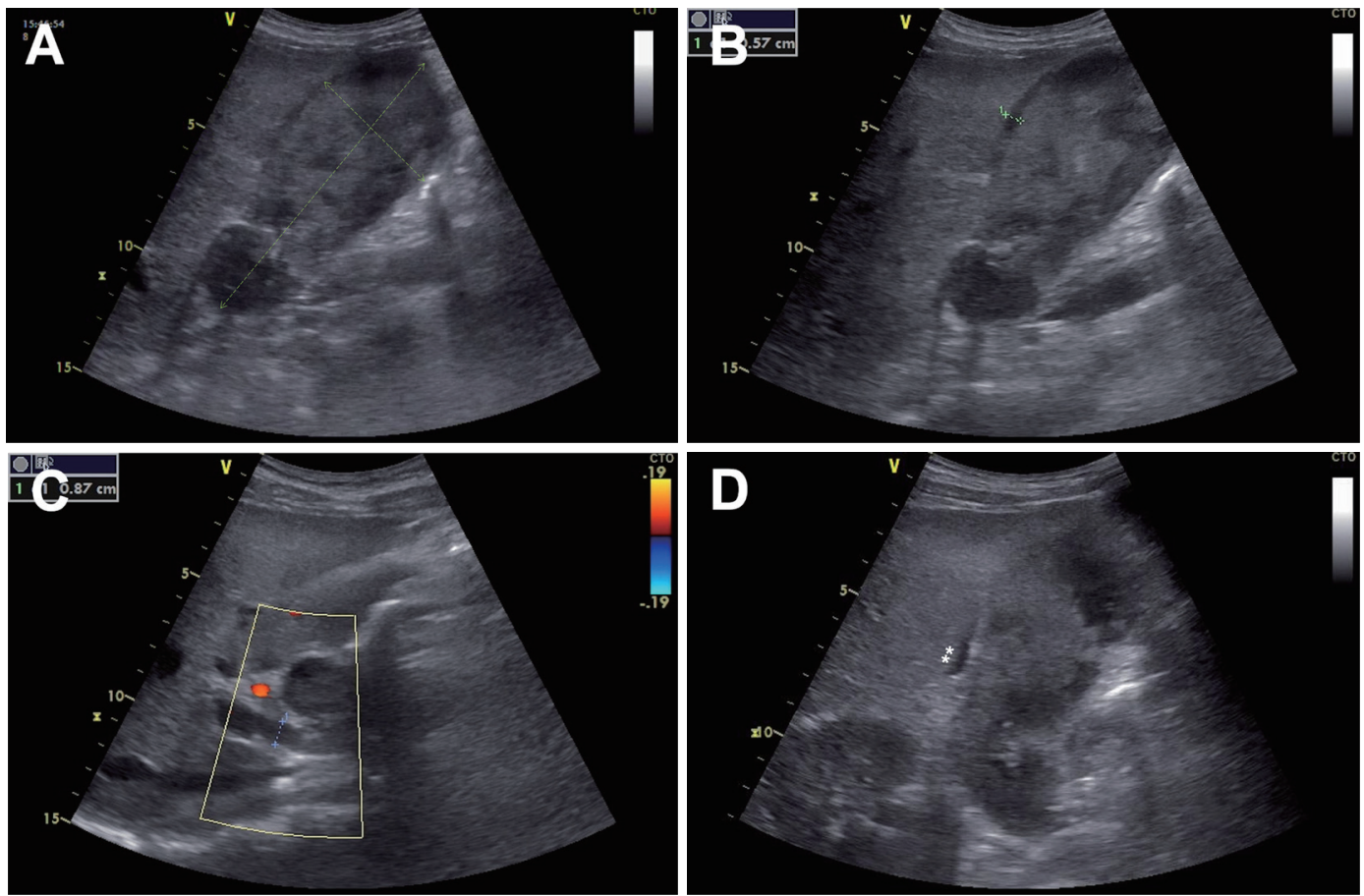

Fig. 2. The findings of the point-of-care ultrasound. A notably large $(118 \times 73 \times 50 \mathrm{~mm})$ gallbladder with hyperechoic hematomas was visualized within the entire lumen (A). The content is nonshadowing and presents as clumps of echoes due to clot accumulations. The wall is $57-\mathrm{mm}$ thick with edema (B). The dilatated proximal common bile duct of $87 \mathrm{~mm}(\mathrm{C})$ and a focal area of pericholecystic fluid (asterisks) is also noted (D). 

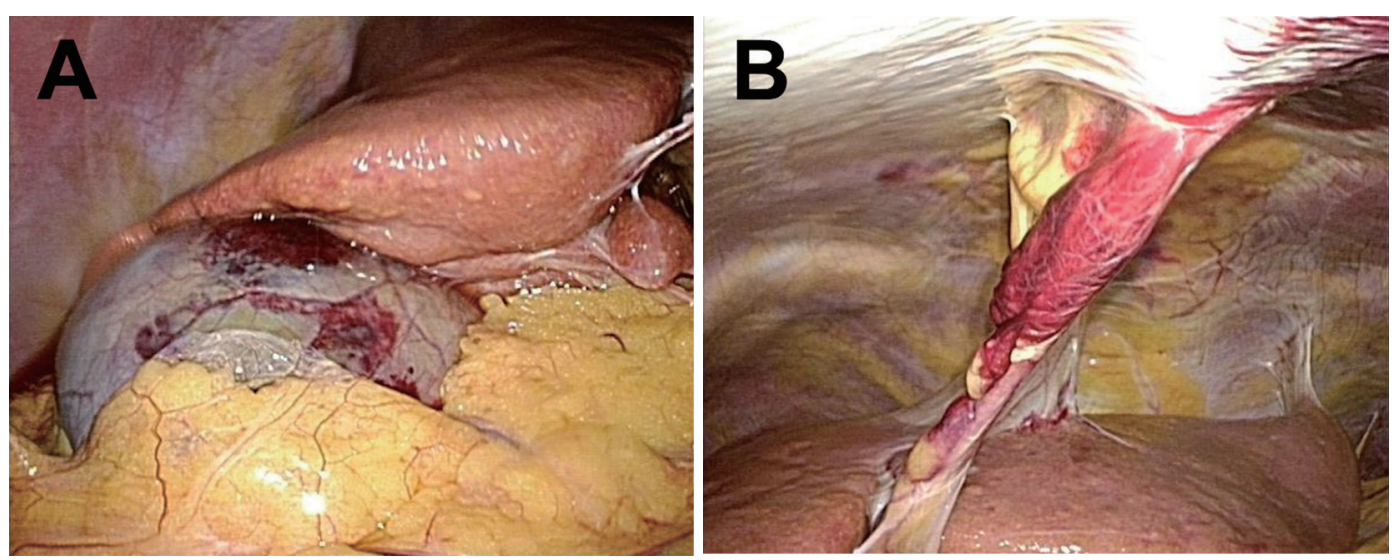

Fig. 3. Intraoperative photographs. The cirrhotic liver parenchyma and the grossly distended and tense gallbladder containing multifocal contusions without a perforation (A) were encountered along with a falciform ligament contusion (B).
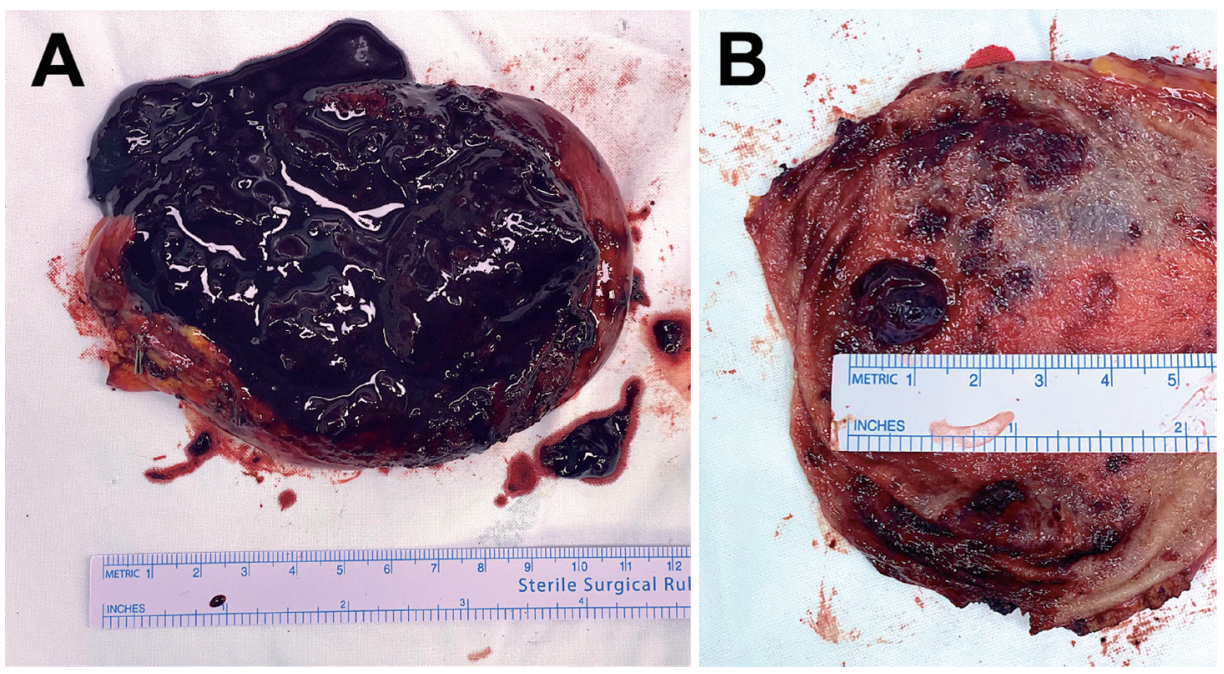

Fig. 4. The gallbladder specimen demonstrates massive clots occupying the lumen (A) with an adenomatous polyp of $15 \mathrm{~mm}(\mathrm{~B})$.

S5 ultrasound (General Electric Healthcare, Chicago, IL, USA) revealed aggravated AHC (Fig. 2). Emergency laparoscopic cholecystectomy was performed based on the patient's stable presentation (Figs. 3 and 4). The laparoscopy showed neither peritoneal fluid collection nor pericholecystic inflammation; thus, the dissection of the Calot's triangle was uneventful. The patient made a complete recovery after the surgery, and the final pathologic diagnosis was transmural hemorrhagic infarction with chronic cholecystitis.

\section{Discussion}

The rarity of the GB injuries from trauma is due to its anatomical location, embedded in the fossa on the liver surface and protected by the ribcage. The patient in the current study had a unique combination of multiple risk factors for traumatic AHC. Severe GB distension, which is caudally exceeding the roof (ribcage), can provide a reason for compression force to breach the protective anatomy. Alcoholism predisposes GB distension by increasing the tone of the sphincter of Oddi and raising the biliary tract pressure (1). One report has proposed that the cirrhotic liver may exacerbate shear forces in the GB fossa by providing a stiff underlay (2). Moreover, the GB polyp and the dilatated common bile duct may give clues to this patient's long history of extrahepatic cholestasis before the accident.

Close follow-up physical examinations and serial imag- 
ing are essential to tailor further AHC management after trauma, and a delay may cause a loss of opportunity for timely and quality treatment. The CT is the most complete test for evaluating cholecystitis (3). However, POCUS is also recommended because it can be frequently performed without the pressure of contrast injection, cost, or time consumption in patients with critical illnesses.

This case demonstrates that laparoscopic cholecystectomy is feasible in treating traumatic AHC with liver cirrhosis in a stable hemodynamic state. The definitive treatment for traumatic AHC is a cholecystectomy while a percutaneous cholecystostomy can be performed in patients with significant comorbidities (4). However, cholecystostomy is not recommended because the hematomas mixed with bile create semisolid lumps that are hardly drained through a pigtail catheter of a small caliber. Recent studies reported that the cholecystectomy in patients with cirrhosis is feasible with low mortality and morbidity, especially in Child's class A (5). The cholecystostomy in patients with cirrhosis who have ascites showed a $14 \%$ risk of adverse events; thus, it is contraindicated (5).

\section{Conflict of interest}

No potential conflict of interest relevant to this article was reported.

\section{References}

1. D'souza C, Bhagavan KR. Isolated gall bladder perforation following a blunt injury in the abdomen. J Clin Diagn Res. 2012;6(8):1409-10.

2. Philipoff AC, Lumsdaine W, Weber DG. Traumatic gallbladder rupture: a patient with multiple risk factors. BMJ Case Rep. 2016;2016:bcr2016216811.

3. Calderón JZR, Chamorro EM, Sanz LI, Merino JCA, Nacenta SB. Hemorrhagic cholecystitis: ultrasound and CT imaging findings-a retrospective case review series. Emerg Radiol. 2021;28(3):613-20.

4. Reens D, Podgorski B. Hemorrhagic cholecystitis: a case of expedited diagnosis by point-of-care ultrasound in the emergency department. J Emerg Med. 2019;57(1):74-6.

5. Pavurala RB, Li D, Porter K, Mansfield SA, Conwell DL, Krishna SG. Percutaneous cholecystostomy-tube for high-risk patients with acute cholecystitis: current practice and implications for future research. Surg Endosc. 2019;33(10):3396-403. 